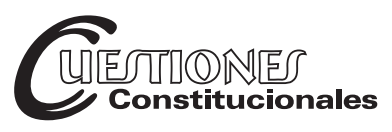

Revista Mexicana de Derecho Constitucional

Núm. 39, julio-diciembre 2018

\title{
Sufragio por Internet: análisis teórico y comparado*
}

\author{
Internet Voting: Theoretical and Comparative Analysis
}

\section{Pablo Marshall Barberan**}

RESUMEN: El presente artículo examina la conveniencia de incorporar el sufragio por Internet desde un punto de vista teórico y de derecho comparado. Parte distinguiendo los conceptos de sufragio electrónico, sufragio por correo y sufragio por Internet. Se analizan los principales atractivos y objeciones que en la literatura comparada se han ofrecido contra el sufragio por Internet. Para esto se sigue de cerca la experiencia con otras formas de votación a distancia, en particular el sufragio por correo que es la forma más común de sufragio en ausencia y presenta ventajas y problemas similares a los del sufragio por Internet. A modo de conclusión se ofrece un balance que sugiere que la introducción de esta clase de medidas, si bien no presentan graves complicaciones para la integridad del proceso eleccionario, tienen el potencial de transformar algunas de las características de la práctica de las elecciones como las conocemos.

Palabras clave: democracia, derecho a sufragio, sufragio electrónico, sufragio por correo, integridad electoral.
ABSTRACT: This paper examines the convenience of incorporating Internet voting from a theoretical points of view and comparative law. It distinguishes the concepts of electronic voting, postal voting and Internet voting. The main arguments for and against Internet voting in the comparative literature are also analysed. I follow the analysis of other forms of absentee voting, in particular voting by mail that is the most common modality of absentee voting and share problems and advantages with Internet voting. It is concluded that the introduction of Internet voting does not present serious problems to the integrity of the electoral process but it has the potential to transform some of the characteristics of the practice of elections as we know them.

Keywords: Democracy, Right to Vote, Electronic Voting, Postal Voting, Electoral Integrity.

* Este artículo se inscribe en el proyecto Fondecyt 11150299 ("Una teoría sobre la función del derecho a sufragio: participación y reconocimiento") del que el autor es investigador principal.

** Profesor de derecho de la Universidad Austral de Chile. Es doctor en derecho por la Universidad de Glasgow y licenciado en derecho por la Universidad de Chile. Editor de la revista Derecho y Crítica Social.pmarshall@uach.cl. 
SUMARIO: I. Introducción. II. Formas de sufragio alternativo: electrónico, por correo e Internet. III. Ventajas del sufragio por correo e Internet. IV. Problemas del sufragio por correo e Internet. V. El significado del sufragio por Internet. VI. Conclusión.

\section{INTRODUCCIÓN}

En varios países se viene acentuando un diagnóstico negativo en relación con los decrecientes niveles de participación electoral, que ha llegado a los niveles más bajos experimentados tras el nuevo auge de la democracia tras la caída del Muro de Berlín. Por ejemplo en Chile, los niveles de participación han caído desde 87\% (1989) hasta 51\% (2013) en las elecciones de diputados. ${ }^{1}$ En estos países, las propuestas que apuntan a facilitar el sufragio para mejorar los índices de participación abundan. Entre ellas se mencionan constantemente la de permitir el sufragio no presencial y la de implementar sistemas de sufragio electrónico.

El sufragio electrónico consiste en un sufragio que es realizado por medio de un dispositivo electrónico. Este concepto incluye el registro electrónico de votos, el conteo electrónico de votos y últimamente canales electrónicos para votar, por ejemplo, por medio de un dispositivo remoto conectado a Internet. Si bien la forma más común de sufragio electrónico implementado en el derecho comparado no implica el uso de esta última modalidad, y consiste en la instalación de máquinas electorales en los locales de votación, en este trabajo me centraré principalmente en la implementación de un sistema de sufragio electrónico de carácter remoto, o sufragio por Internet como lo denominaré.

Me interesa abordar la interacción entre el sufragio electrónico y otra modalidad de sufragio que también ha recibido importante atención en el derecho comparado durante las últimas décadas. Me refiero al sufragio en ausencia. En conjunto con el sufragio anticipado (early voting), el sufragio en ausencia (absentee voting), que puede ser definido como una forma de sufragar que prescinde de la concurrencia del elector al recinto de votación en el día de la elección, es la forma en que las reformas electorales

PNUD, Participación electoral: Chile en perspectiva comparada 1990-2016, Santiago, Programa de las Naciones Unidas para la Democracia, 2016. 
han intentado hacer más conveniente (convenience voting) o amistosa la convocatoria electoral. La forma más común de sufragio en ausencia es el sufragio por correo (voting by mail), por lo que la discusión de esta modalidad de sufragio será considerada con cierto detalle, en la medida que el sufragio por Internet se enfrente a similares problemas, teniendo ventajas también similares.

El objeto central del presente análisis es el sufragio por Internet, que es una modalidad combinada que incluye el uso de mecanismos tecnológicos (computadores y teléfonos inteligentes) y un sufragio que no requiere la presencia del votante en el recinto electoral, sino que puede ser realizado desde un lugar diferente (hogar, lugar de trabajo, vía pública). Pese a lo atractivo que resulta para muchos en nuestros días pensar en sufragar por un medio remoto, en la medida que el sufragio por Internet es una modalidad que no ha sido implementada en la práctica, salvo muy limitadas excepciones, muchas de las consideraciones teóricas y prácticas que se realizan en este trabajo son formuladas por aproximación y obtenidas de las discusiones sobre sufragio electrónico y sufragio por correo.

Este tratamiento del sufragio por Internet permitirá por un lado adelantar algunas hipótesis sobre su conveniencia como forma alternativa de votación y con respecto a su implementación institucional. Sin embargo, también se consideran ciertos rasgos que usualmente creemos que pertenecen por defecto a las elecciones, como su simultaneidad, su ocurrencia en un espacio público o el carácter secreto que reviste el sufragio, y que podrían verse cuestionados por la implementación del sufragio por Internet. Ello, considero, podría tener una influencia más que anecdótica en la forma en que comprendemos el rol de las elecciones y el sufragio en el contexto de la democracia representativa en los tiempos de la sociedad de la información.

Una nota aclaratoria es necesaria antes de proceder. El análisis que se lleva a cabo en este trabajo pretende servir como un insumo general para el análisis del sufragio por Internet. Los razonamientos y conclusiones son de tipo abstracto y su aplicación a una sociedad en particular requiere, por lo tanto, de la consideración de características concretas de cada Estado en cuestión, como lo son la cultura política, el diseño institucional o la trayectoria democrática. El estudio pormenorizado de esos factores particulares debe proceder en estudios de casos que, no obstante, puede ser beneficiado por las observaciones generales que serán planteadas aquí. 
El trabajo se estructura de la siguiente manera. En la sección I se expone el funcionamiento y las modalidades del sufragio electrónico, el sufragio por correo y el sufragio por Internet. En la sección II se revisan las ventajas y en la sección III los problemas que las modalidades remotas de votación enfrentan en la teoría y en la práctica. Por último, en la sección IV se evalúa el significado que la implementación del sufragio por Internet podría tener para la comprensión que tenemos de nuestra práctica electoral.

\section{FoRMAS DE SUFRAGIO ALTERNATIVO: ELECTRÓNICO, POR CORREO E INTERNET}

Esta sección describe tres formas de sufragio que difieren del tradicional sufragio presencial en papel. Se trata de dos modalidades que ya tienen cierta aplicación práctica en las democracias occidentales y sobre las que se cuenta con mayor información: el sufragio presencial electrónico y sufragio en ausencia por correo, y de una tercera que consiste en una intersección entre ellas, pero sobre la que se posee poca información dado su incipiente estado de desarrollo, el sufragio en ausencia por Internet.

\section{Sufragio electrónico}

El sufragio electrónico se encuentra en la intersección entre el proceso democrático y el uso de las nuevas tecnologías en lo que ha sido denominado como democracia electrónica o e-democracy. ${ }^{2}$ La democracia electrónica ha involucrado, por un lado, una serie de reformas que utilizando los nuevos medios tecnológicos disponibles han intentado entregar soluciones novedosas a problemas democráticos actuales. Por otro lado, sin embargo, la democracia electrónica se presenta como un nuevo paradigma al que la forma democrática debe adaptarse si pretende sobrevivir en una sociedad marcada por la globalización, el flujo permanente de la información y la dependencia cada vez más intensa de medios tecnológicos para el funcionamiento de la sociedad y con masiva influencia en la vida diaria de cada

\footnotetext{
2 Kersting, Norbert y Baldersheim, Harald, "Electronic Voting and Democratic Issues: An Introduction", Electronic Voting and Democracy, Londres, Palgrave Macmillan, 2004, pp. 4 y 5.
} 
uno de sus integrantes. ${ }^{3}$ La incorporación de referéndums electrónicos, encuestas deliberativas y nuevas formas de democracia directa se cuentan entre sus implementaciones más atractivas en esta línea. ${ }^{4}$ Volveré sobre este asunto de la capacidad transformadora de la tecnología en la sección final del artículo. Lo importante de constatar aquí es que frente a la tensión entre la facilitación tecnológica y la revolución que nos dirige a una nueva era digital, la incorporación de la tecnología en los procesos electorales ha sido una de las pocas esferas que evolucionado lenta y cautelosamente. ${ }^{5}$

El uso de máquinas electrónicas de votación tiene una larga tradición en los Estados Unidos. Además, se ha implementado de manera permanente en países como India, Brasil, Paraguay, Venezuela y Filipinas. En general, esta modalidad de votación ha buscado superar problemas en la organización y la administración de las elecciones en países con grandes extensiones geográficas, problemas como el mantener la confianza en la democracia aumentando la rapidez en el conteo de sufragios y el diseño de estrategias para incentivar una mayor participación electoral. No obstante su creciente popularidad, lo que hace presumir que su implementación ha cumplido con su objetivo general de mejorar la organización y la administración electoral, existen experiencias negativas como la elección general de Holanda en 2006 y California en 2004, que hicieron que las autoridades decidieran volver a las modalidades tradicionales de sufragio. ${ }^{6}$ Problemas de confianza pública en esta modalidad de votación llevaron al abandono de los proyectos de digitalización electoral.

La dimensión tecnológica de los sistemas de votación electrónica generan un conjunto de cuestiones técnicas y procedimentales que deben examinarse detalladamente, y que van desde el diseño y ejecución del software a usar, la adquisición del hardware en casos de voto elec-

3 Belmonte Martín, Irene, “Pueden ser las nuevas tecnologías un instrumento para reforzar las democracias?", Revista de la Facultad de Ciencias Sociales y Jurídicas de Elche, vol. 1, núm. 2, 2007, pp. 1-23.

4 Oostveen, Anne-Marie y Van den Besselaar, Peter, "Internet Voting Technologies and Civic Participation: the Users' Perspective", Javnost-The Public, vol. 11, núm. 1, 2004, p. 63.

5 Martínez Dalmau, Rubén, “Aspectos diferenciales del uso del voto electrónico en los procesos electorales y en los órganos colegiados", Anuario de Derecho Parlamentario, vol. 25, 2011, pp. 229-245.

6 Tula, María Inés, "Democracia, elecciones y nuevas tecnologías. El voto electrónico", Revista Mexicana de Análisis Político y Administración Pública, vol. 1, núm. 2, 2012, pp. 9-21. 
trónico presencial, pasando por los mecanismos de seguridad informática que resguarden el sistema contra accesos ilegítimos, procedimientos para verificar su correcto funcionamiento, la logística de la organización y la transmisión de la información; llegando a incluir la capacitación de la administración electoral y la ciudadanía en la manipulación de estos sistemas. La literatura especializada en estos numerosos aspectos ha crecido considerablemente en la medida que sistemas electrónicos son cada vez más usados. ${ }^{7}$ Sin embargo, la consideración in extenso de las discusiones que se han dado en torno a ellas, que son de una extensión y complejidad técnica considerables, está fuera del alcance de este trabajo. ${ }^{8}$

Uno de los aspectos que sí es importante mencionar aquí, es el rol que el acceso a Internet tiene en la implementación del voto electrónico. Este uso puede involucrar al menos tres situaciones. Primero, en una votación presencial, el elector vota usando una máquina electrónica de votación que a través de Internet (o intranet con la finalidad de prevenir ataques informáticos externos) comunica la información a la autoridad electoral local, regional o central. Segundo, en un lugar público el elector usa una computadora especialmente habilitada a la cual tiene acceso usando un instrumento electrónico de autenticación (como una tarjeta o una huella digital). Esta modalidad se conoce por la literatura especializada como kiosk voting. Tercero, situado en cualquier lugar, el elector usa para votar cualquier computador o teléfono móvil usando un software u otro medio de autenticación. ${ }^{9} \mathrm{La}$ principal diferencia entre la primera situación y las dos últimas es la existencia de un entorno oficialmente controlado que limita la posibilidad de vulneraciones a la seguridad y confiabilidad del proceso.

\footnotetext{
7 Idem.

8 Véase Krimmer, Robert, Electronic Voting 2006, GI Lecture Notes in Informatics, Bonn, 2006, p. 86; Celeste, Richard et al., Asking the Right Questions About Electronic Voting, Washington, National Academies Press, 2006; Tuesta, Fernando, "El voto electrónico", Elecciones, vol. 3, 2004, pp. 55-80; Thompson, José, "La experiencia reciente del voto electrónico en América Latina: avances y perspectivas", Revista de Derecho Electoral, vol. 7, 2009, pp. 9 y ss.; Reyes, Guillermo, El voto electrónico y por Internet como refuerzo de la confiabilidad de los sistemas electorales, tesis doctoral, Universidad Complutense de Madrid, Facultad de Derecho, 2015.

9 Kersting, Norbert y Baldersheim, Harald, "Electronic Voting and...", cit., p. 7; Oostveen, Anne-Marie y Van den Besselaar, Peter, "Internet Voting Technologies...,", cit., p. 63 .
} 


\section{Sufragio por correo}

El sufragio en ausencia es parte de una tendencia de reformas electorales que han intentado hacer el voto más conveniente y así incrementar la participación electoral. La idea principal detrás del sufragio en ausencia es ofrecer al elector una opción para poder sufragar sin tener que concurrir a la mesa de votación en el día de la elección. Se asume que esto podría tener una influencia en aquellas personas que no pueden o no se sienten motivadas por desplazarse al local de votación. Es así como puede constituirse en una solución a dos problemas diferentes. Por un lado, busca habilitar a ciertas personas excluidas por diferentes razones de la posibilidad de concurrir a sufragar. Enfermos postrados, personas con discapacidad motriz, personas que habitan lugares remotos o que se encuentran lejos de su local de votación o en el extranjero, son algunos ejemplos de las personas afectadas por el primer problema. Por otro lado, el sufragio en ausencia busca rebajar la carga que supone sufragar para el elector haciendo esta actividad más conveniente y por tanto motivando a personas que de otra manera sería difícil que concurrieran a votar.

El sufragio en ausencia es bastante popular en los regímenes electorales comparados y su forma más común es la del sufragio por correo. Este último consiste en una modalidad en que el votante recibe una papeleta de votación por correo y luego tiene un periodo de tiempo para devolverlo también por correo antes del día de la elección. ${ }^{10}$ Fue implementado, originalmente, para permitir votar a los militares que estaban en servicio fuera de su localidad de votación y se extendió a un gran número de casos en que personas lo usan por razones religiosas (cuando la elección se realiza en un día sagrado), por estar el elector hospitalizado, en prisión o residiendo en el extranjero. En su origen, el sufragio en ausencia fue introducido como un régimen excepcional al que podían acceder sólo las personas afectadas por una imposibilidad como las anotadas. Ellos debían manifestar una excusa que los habilitara a acceder al beneficio del sufragio por correo. Sin embargo, con el tiempo éste fue convirtiéndose en un derecho disponible para todos los ciudadanos sin necesidad de presentar alguna excusa. Ello se debió a que se buscaba, de esta manera, incrementar la participación por medio de reducir el "costo de transacción" de sufragar. El voto por co-

10 Qvortrup, Matt, "First Past the Postman: Voting by Mail in Comparative Perspective”, The Political Quarterly, vol. 76, núm. 3, 2005, p. 414. 
rreo, así, fue ampliando su funcionalidad, de una modalidad que permitía incluir a los excluidos por el sistema de voto presencial, a una modalidad de sufragio que permite rebajar la carga que significa concurrir al local de votación. ${ }^{11}$

Las principales variantes del sufragio por correo, que son usadas en numerosos países, son las siguientes: 1) sistema de sufragio por correo generalizado: este sistema, usado en algunas elecciones locales en los Estados Unidos, prescinde totalmente del voto presencial, convirtiendo el sufragio por correo en la única forma de participar electoralmente; 2) sufragio por correo sin excusa: todos los electores pueden solicitar la votación en ausencia para lo cual una papeleta de votación les es enviada por correo con más de un mes de anticipación, la que deben devolver por la misma vía, 3) sufragio por correo con excusa: en este caso los solicitantes deben demostrar la circunstancia de estar en un caso en que el voto en ausencia es permitido, por ejemplo, en el servicio militar, en el extranjero o en el hospital. $^{12}$

Sin embargo, no sólo es común que la legislación contemple estas modalidades de votación sino que además su uso ha aumentado y son muy populares entre los electores, llegando a involucrar un gran porcentaje del electorado en su uso. Por ejemplo, en la elección de 2006 en los Estados Unidos constituyó un $25 \%$ de los votos emitidos, pero no depositados en los recintos de votación. ${ }^{13}$ En las elecciones de 2010 en el Reino Unido y Australia, esa cifra se situó en torno al 20\%.14

La principal discusión acerca del sufragio en ausencia en general y del sufragio por correo en particular ha girado en torno a si los niveles de participación pueden incrementarse mediante su utilización sin por ello amenazar los niveles de integridad electoral deseados. La preocupación por la manera en que esta modalidad de sufragio puede ser problemática se ha concretado en dos críticas. Por un lado, se argumenta que las formas no presenciales de sufragio son vulnerables tanto al fraude electoral como a la coacción por parte de terceros. Por otro lado, se ha señalado, en

11 Fortier, John, Absentee and Early Voting. Trends, Promises, and Perils, Washington, AEI Press, 2006, pp. 7-17.

12 Gronke, Paul et al., "Convenience Voting”, Annu. Rev. Polit. Sci., vol. 11, 2008, p. 441 .

13 Ibidem, p. 440; Fortier, John, Absentee and Early..., cit., pp. 18-39.

14 Orr, Graeme, Ritual and Rhythm in Electoral Systems: a Comparative Legal Account, Londres, Routledge, 2016, p. 53. 
particular respecto del sufragio por correo, que es más proclive a errores en la votación, el traslado del sufragio y el procesamiento de éste, por lo que acarrearía una tasa más alta de sufragios no procesados por el sistema como votos válidamente emitidos. Volveremos sobre estos problemas en la sección siguiente.

\section{El sufragio por Internet}

El sufragio electrónico remoto o más comúnmente conocido como sufragio por Internet, consiste en una modalidad de votación en que el votante marca su preferencia mediante el uso de un dispositivo tecnológico conectado al Internet. El sufragio por Internet es una forma de sufragio en ausencia y de esta manera reproduce algunas de las características del sufragio por correo. Sin embargo, el uso de la tecnología electrónica implica, para esta modalidad, una nueva serie de aspectos que lo diferencian de la categoría previa, relacionados por ejemplo con la vulnerabilidad de las elecciones conducidas por Internet ante ataques informáticos o la facilidad e inmediatez con que esta modalidad permite transmitir la preferencia en relación con el sufragio por correo. Todos estos aspectos serán debidamente considerados en las siguientes secciones.

La introducción del sufragio por Internet ha sido consecuencia del auge que todo tipo de actividades llevadas por este medio ha tenido en las últimas dos décadas. Las votaciones por Internet parecen muy populares en nuestros días, proliferando en ámbitos como las empresas, las organizaciones no gubernamentales o los cuerpos estudiantiles y universidades. Sin embargo, ellas no parecen tener acogida en lo que respecta a las elecciones de autoridades públicas. Hay, evidentemente, impedimentos legales y constitucionales para ello, sin embargo, los más importantes obstáculos para ello parecen ser los reparos que se han manifestado respecto a su capacidad para mantener el secreto respecto a su voto individual y mantener la integridad del proceso electoral en general. No obstante esto, el sufragio por Internet ha sido utilizado con el afán de verificar su funcionamiento en varios países. Ese ha sido el caso en elecciones locales, generales y comunitarias de ciertos estados de los Estados Unidos, el Reino Unido, Suiza, Noruega, India y Canadá. En todos estos casos, pese a tratarse de pilotos, la implementación del sufragio por Internet sigue en consideración. Por otro lado, el sufragio por Internet ha sido implementado como una plata- 
forma permanente para ejercer el sufragio sólo en un país hasta ahora: las elecciones estonias han contado con la posibilidad de votar por Internet desde 2005 en todos los niveles (local, general y comunitario) y tras 10 años de práctica, y ocho elecciones realizadas, el sufragio por Internet ha correspondido al 30\% de los votos emitidos en la elección de 2015, lo que significa un considerable incremento respecto al escaso $2 \%$ que votó en las elecciones de $2005 .{ }^{15}$ Finalmente, existen países donde el sufragio por Internet ha sido implementado de manera permanente pero sólo se encuentra disponible para ciertos grupos de personas: en el caso de Francia para los ciudadanos viviendo fuera del país y en los Estados Unidos para los militares que se encuentran fuera del país en operaciones armadas.

La descripción del modelo implementado en Estonia puede iluminar cómo funciona el sufragio por Internet en la práctica. En lo que respecta al proceso de autenticación, el votante hace uso de su tarjeta nacional de identificación, lo que sumado a la combinación de dos números secretos (uno para acceder al voto y otro para sufragar) hacen que la seguridad contra la suplantación sea considerablemente estricta. Desde 2011 también se implementó un sistema de votación por medio de teléfonos inteligentes con tarjetas SIM especiales. Los votantes utilizan un software especial que deben descargar, el que les permite además verificar si su voto ha sido recibido por el sistema. La votación por Internet está abierta por un periodo de tiempo que ha variado de 3 a 7 días antes del día de la elección. Como puede verse, el procedimiento no debería ser complicado para personas con experiencia en realizar sus transacciones bancarias por Internet. ${ }^{16}$

\section{VENTAJAS DEL SUFRAGIO POR CORREO E INTERNET}

Existen una serie de ventajas y problemas que se han discutido en relación con las formas no presenciales de votación. Esta discusión ha estado centrada en el sufragio por correo dado que es la forma más extendida de sufragio en ausencia. En esta sección y la siguiente me gustaría considerar estas ventajas y problemas a la luz de la evidencia disponible y a la dis-

15 Vassil, Kristjan et al., "The Diffusion of Internet Voting. Usage Patterns of Internet Voting in Estonia between 2005 and 2015”, Government Information Quarterly, vol. 33, núm. 3, 2016, pp. 453-459.

16 Ibidem, p. 454. 
cusión teórica llevada a cabo en la literatura especializada y evaluar estos aspectos a la luz de las alternativas que el sufragio por Internet puede ofrecer.

\section{Aumento de la participación, facilidad e inclusión}

Como ya se ha señalado, la principal ventaja que es reclamada por las modalidades de sufragio en ausencia, y el principal argumento esgrimido para su implementación en el derecho comparado, es que permite incrementar la participación electoral. El razonamiento consiste en afirmar que concurrir al lugar de votación es un costo de transacción para el votante y que mediante la eliminación de dicho costo la participación de personas que se veían disuadidas por él aumentará. ${ }^{17}$ Puesto de una manera más informal: "Si los ciudadanos no vienen a las urnas, por qué no llevar las urnas más cerca de los ciudadanos". ${ }^{18}$ Además del aumento en la participación, se considera que las modalidades de sufragio en ausencia cumplen con el rol de incluir a personas a los que concurrir presencialmente a votar se hace muy costoso o imposible. Entre estas personas, ya se ha mencionado, pueden contarse las personas con discapacidades, las personas que viven en el extranjero o madres que no tienen con quien dejar a sus hijos. Pueden denominarse a estos dos objetivos el objetivo facilitador y el objetivo inclusivo del sufragio en ausencia. La satisfacción de estos objetivos descansa sobre la premisa de que el ejercicio del sufragio sea efectivamente más fácil y accesible. Parte de lo que hace atractivo, en este sentido, al sufragio en ausencia, además de realizarse desde un lugar más conveniente para el votante, es que normalmente entrega a éstos la oportunidad de sufragar en cualquier momento, lo que constituye una importante diferencia respecto

17 Qvortrup, Matt, "First Past the Postman...", cit., p. 415; Bochsler, Daniel, "Can Internet Voting Increase Political Participation?", trabajo presentado en el Seminario Internet and Voting, Fiesole, 2010, pp. 3 y 4, disponible en: http://www.eui.eu/Projects/ EuDO-PublicOpinion/Documents/bochslere-voteeui2010.pdf (consultada el 7 de marzo de 2017).

18 Norris, Pippa, "E-Voting as the Magic Ballot for European Parliamentary Elections? Evaluating e-Voting in the Light of Experiments in UK Local Elections", en Trechsel, Alexander y Mendez, Fernando (edits.), The European Union and e-Voting. Addressing the European Parliament's Internet Voting Challenge, Londres, Routledge, 2005, p. 60 . 
del sufragio presencial, que sólo puede ser ejercido en un día y horario predeterminado, lo cual puede generar inconvenientes.

La evidencia sobre el efecto del sufragio por correo en la participación electoral es en general no concluyente y casi todos los estudios realizados consideran que el incremento en la participación producida por su introducción es marginal. ${ }^{19}$ En general la literatura especializada vincula la baja participación electoral a factores como el interés individual y los niveles de información sobre política, la posición social del votante, la competitividad de la elección y el contexto de la campaña electoral, ${ }^{20}$ lo que no puede ser solucionado mediante la facilitación del acto de sufragar.

Aunque la evidencia no es concluyente a este respecto, existen otras variables que son de gran interés y que deben consideradas conjuntamente. La primera, y la más importante, es la variable social. ¿Cuáles son los sectores sociales que aumentan su participación? Si en un comienzo el sufragio por correo tenía la finalidad de beneficiar a los grupos que no podían acceder al sufragio presencial, esto es, servía para un objetivo inclusivo, la evidencia encontrada en los estudios llevados a cabo en Estados Unidos, Australia y el Reino Unido indica que estos grupos no han utilizado este mecanismo en una proporción más importante que el resto de la población y que el incremento general puede deberse al incremento entre grupos de la población que son más propensos a concurrir a votar. Esto quiere decir, que no lograría satisfacer tampoco su objetivo inclusivo. Por otro lado, se ha acusado al sufragio por correo de aumentar la brecha entre la participación de los sectores altos y medio-altos en detrimento de los sectores bajos y medio-bajos. La evidencia muestra que el aumento general en la participación se ha concentrado en un aumento focalizado en los primeros grupos socioeconómicos sin afectar la participación de los segundos. ${ }^{21}$ Una segunda variable a considerar en conjunto con el aumento de participación es si tal aumento incluye efectivamente a nuevos votantes. Se ha encontrado evidencia en estudios conducidos en los Estados Unidos que el sufragio

19 Qvortrup, Matt, "First Past the Postman...", cit., p. 417; Gronke, Paul et al., "Convenience...", cit., pp. 442 y 443.

20 Gronke, Paul et al., "Convenience Voting", cit., p. 442.

21 Alvarez, R. Michael y Nagler, Jonathan, "Likely Consequences of Internet Voting for Political Representation”, Loy. LAL Rev., vol. 34, 2000, pp. 1115-1153; Qvortrup, Matt, "First Past the Postman...", cit., pp. 417 y 418; Gronke, Paul et al., "Convenience Voting", cit., pp. 443, 447; Stewart, Charles, "Adding up the Costs and Benefits of Voting by Mail”, Election Law Journal, vol. 10, núm. 3, 2011, p. 301. 
por correo no tiene la capacidad de persuadir a nuevos votantes, sino que su eficacia se ve limitada a conservar a votantes irregulares, para que sigan votando en elecciones en que otros factores los llevarían a no participar. $^{22}$

Cuando se analiza la evidencia disponible en relación con el sufragio por Internet, las cosas no cambian demasiado. No existe evidencia concluyente de que su implementación implicaría un aumento relevante de la participación, pero sí existe evidencia de que tal incremento, de producirse, se enfocaría en ciertos grupos, ${ }^{23}$ aunque esa evidencia no coincide con la correspondiente al sistema estonio, donde no se observaron vinculaciones entre el sufragio por Internet y perfiles basados en género, ingreso y preferencias políticas, pese a coincidir en lo anecdótico con el aumento de la participación general. ${ }^{24}$

Pese a no haber evidencia de ello, debe notarse que los sistemas de sufragio por Internet disponibles deberían tener prioridad sobre el sufragio por correo cuando se trata de perseguir los objetivos de facilitación e inclusión. Por un lado, éstos son considerablemente menos engorrosos y demandantes en términos de tiempo y desplazamientos que las modalidades de sufragio por correo, lo que podría justificar una preferencia del primero sobre el segundo, cuando se trata de priorizar el objetivo facilitador de una reforma. Por ejemplo, con el sufragio por Internet no es siquiera necesario visitar el correo para depositar el voto y puede constituir un incentivo para participar cuando un clima lluvioso, largas esperas, trabajo que hacer o el costo del transporte tienden a disuadir a un votante indeciso. Esta ventaja, sin embargo, no parece considerable y puede revertirse en casos como el suizo, en que el proceso para la obtención del material para sufragar por Internet era más complicado que sufragar por correo. ${ }^{25}$ Por otro lado, en

22 Gronke, Paul et al., "Convenience Voting”, cit., p. 444.

23 Alvarez, R. Michael y Nagler, Jonathan, "Likely Consequences of...", cit.; Norris, Pippa, "E-Voting as the...", cit., pp. 84 y 85; Bochsler, Daniel, "Can Internet Voting...", cit.; Beroggi, Giampiero, "Internet Voting: An Empirical Evaluation", IEEE Computer, vol. 47, núm. 4, 2014, p. 29; Hall, Thad, "Internet Voting: the State of the Debate", en Coleman, Stephen y Freelon, Deen (eds.), Handbook of Digital Politics, Elgar Publishing, 2015, p. 104; Saglie, Jo y Bock Segaard, Signe, "Internet Voting and the Secret Ballot in Norway: Principles and Popular Understandings", Journal of Elections, Public Opinion and Parties, vol. 26, núm. 2, 2016, pp. 160 y 161.

24 Álvarez, R. Michael et al., "Internet Voting in Comparative Perspective: the Case of Estonia”, Political Science \& Politics, vol. 42, núm 3, 2009, pp. 497-505; Vassil, Kristjan et al., "The Diffusion of...", cit., pp. 456, 458.

25 Beroggi, Giampiero, “Internet Voting: An...”, cit., p. 29. 
lo que respecta a su objetivo inclusivo el sufragio por Internet debería servir mejor a dichas personas que se encuentran lejos de los centros de votación y que tienen dificultades para desplazarse. En este aspecto el uso de la tecnología podría significar una mejora en las condiciones para una participación que atiende a las diferencias de posiciones en que los votantes se encuentran. ${ }^{26}$ La tecnología electrónica podría permitir, por ejemplo, que personas con discapacidades visuales pudieran usar sus propios mecanismos tecnológicos y softwares especializados a la hora de ejercer su sufragio.

Esas no son, sin embargo, las principales ventajas que los defensores del sufragio por Internet tienen en mente. Su principal ventaja estaría dada por su capacidad de incrementar la participación electoral de la gente joven que está familiarizada con el uso de Internet para toda clase de asuntos o aquella que no obstante no ser tan joven está familiarizada con el uso de la tecnología. Pero sus ventajas no son a toda prueba. Como se señalara, la posibilidad de votar por Internet puede no ser una circunstancia suficiente para persuadir a personas que están desilusionadas de la política o simplemente carecen de interés por ésta. La evidencia con que se cuenta de las elecciones con sufragio por Internet en Estonia indican que la conexión entre sufragio por Internet y votantes jóvenes no tiene respaldo en la práctica. Factores como el manejo del idioma en que el software está programado, la confianza en el sistema electrónico de votación o los niveles de competencia en el uso del Internet, aparecen con mayor relevancia en cuanto indicadores de participación. ${ }^{27} \mathrm{Si}$ bien los jóvenes pueden expresar una percepción más positiva hacia el sufragio por Internet que otros ciudadanos, esto no necesariamente los hace más propensos a sufragar por esa o cualquier otra vía. ${ }^{28}$

Una nota aparte se requiere para la comparación entre el sufragio por Internet y el sufragio por correo en lo que se refiere a los votos que no son finalmente contados. Conforme a Stewart, uno de los costos hundidos del sufragio por correo es la pérdida de votos. ${ }^{29}$ Esto se explicaría porque muchas papeletas de votación se pierden en la búsqueda de los electores, por ejemplo, cuando éstos han cambiado su domicilio. Otros tantos votos,

\footnotetext{
26 Vassil, Kristjan et al, "The Diffusion of Internet...", cit., p. 458.

27 Ibidem, p. 456.

28 Hall, Thad, "Internet Voting: the...", cit., pp. 106 y 107.

29 Stewart, Charles, "Adding up the Costs...", cit.
} 
se pierden en la dirección opuesta, dado que no llegan a las autoridades electorales antes del día de la elección. Finalmente, el sufragio por correo, señala Stewart, incrementa el número de votos nulos respecto a los votos realizados presencialmente frente a un oficial que le explica directamente al elector cómo votar y que puede atender todas las dudas que le surjan. Los datos que aporta no son insignificantes, dado que, en la elección presidencial de los Estados Unidos de 2008, 4 millones de votos fueron solicitados, pero no recibidos, 3 millones fueron recibidos pero devueltos para el conteo y casi un millón fueron devueltos pero considerados nulos. ${ }^{30}$

En relación con este tipo de problemas, el sufragio por Internet tiene varias ventajas que permitirían capitalizar un alto número de votos que se pierden por el mal funcionamiento del sistema de sufragio por correo. Primero, no necesita largos tiempos de traslado como en el caso del sufragio por correo. Este problema puede incrementarse en países que no cuentan con un buen y confiable sistema de correos, que garantice tanto que los votos serán entregados en los plazos requeridos. ${ }^{31}$ La inmediatez de la comunicación por Internet solucionaría el problema del retraso que afecta la posibilidad de sufragar de algunos ciudadanos. Segundo, el sistema de sufragio por Internet, no obstante ser vulnerable a ataques informáticos, no es vulnerable la pérdida de votos producto del mal funcionamiento del sistema de correo. Tercero, el sistema de sufragio por Internet debería reducir considerablemente el número de votos que no son contados producto de un error del votante. Esto, en la medida que, a diferencia del sufragio por correo, los softwares de votación pueden configurarse de manera de volverse inmunes a la anulación del sufragio y permiten alertar al votante de la falta de un requisito, paso o autenticación faltante. ${ }^{32}$ Por supuesto que esta ventaja también puede tener aspectos negativos. En la medida que la interacción con el sistema electrónico de votación sólo permite la expresión de preferencias válidas, se produce una reducción de la discrecionalidad individual en la manipulación del voto y se elimina la posibilidad, por ejemplo, de reivindicar un voto nulo como un voto de protesta. ${ }^{33} \mathrm{Sin}$

30 Gronke, Paul et al., "Convenience...", cit., p. 448; Hall, Thad, "Internet Voting: the...", cit., p. 105.

31 Orr, Graeme, Ritual and Rhythm..., cit., p. 57.

32 Hall, Thad, "Internet Voting: the...", cit., pp. 105 y 108.

33 Martínez Dalmau, Rubén, “Aspectos diferenciales del...”, cit., p. 237. 
embargo, esta desventaja puede solucionarse fácilmente incorporando la posibilidad de manifestar un voto de rechazo o un voto en blanco.

El sufragio por Internet, sin embargo, presenta una desventaja estructural respecto del sufragio por correo. La brecha digital es la expresión que hace referencia al desigual acceso a las nuevas tecnologías que afecta a parte de la población por diversas razones sociales, como son las etarias, culturales o económicas. ${ }^{34}$ Ésta podría constituir una razón para que el sufragio por Internet no genere, en tanto la brecha se mantenga, el incremento en la participación deseada. ${ }^{35}$ En la medida que se mantengan los mecanismos tradicionales de votación y no se remplacen éstos por una elección llevada a cabo completamente por Internet, la brecha digital no debería ser preocupante como un factor de declive de la participación. Junto a esto debe considerarse que es previsible que la brecha digital desaparezca en un plazo no demasiado largo en la medida que la dimensión virtual de la vida social se ha incrementado exponencialmente durante la última década y promete seguir haciéndolo.

\section{Rebaja de los costos económicos y operativos de las elecciones}

Aunque no es la principal ventaja que se menciona del sufragio en ausencia, la rebaja de costos económicos ha sido reconocida como un efecto beneficioso que tendría su implementación. Esta ventaja se acentuaría allí donde las elecciones sean realizadas prescindiendo totalmente de locales de votación y descansando únicamente en la participación de los electores por medio de un mecanismo electrónico remoto. Por ejemplo, la experiencia del sistema electoral del estado de Oregón en los Estados Unidos, muestra que, convirtiendo su sistema de votación por correo en la única forma de participar en la elección, las autoridades bajaron el costo de cada voto de 4.33 a 1.24 dólares. ${ }^{36}$ Sin embargo, la conclusión de una revisión sistemática de la información disponible indica que no hay suficiente base para hablar de una rebaja de costos económicos del sufragio en ausencia. ${ }^{37}$

34 Norris, Pippa, Digital Divide: Civic Engagement, Information Poverty, and the Internet Worldwide, Cambridge, Cambridge University Press, 2001.

35 Alvarez, R. Michael y Nagler, Jonathan, "Likely Consequences of...", cit., p.

1119; Kersting, Norbert y Baldersheim, Harald, "Electronic Voting and...", cit.

36 Qvortrup, Matt, "First Past the Postman...", cit., p. 415.

37 Gronke, Paul et al., "Convenience Voting...", cit., p. 449. 
Entre sus defensores, existe la creencia de que el sufragio por Internet producirá una rebaja de costos mayor que el sufragio por correo, pero no existe evidencia al respecto. Si bien un sistema por Internet podría implicar una rebaja considerable de los costos asociados a la administración electoral, esto no debe confundirse, sin embargo, con los costos iniciales de diseño, adquisición e implementación de un nuevo sistema de votación que descansa en dispositivos tecnológicos, los que pueden ser muy costosos. ${ }^{38}$ Aún más, allí donde las elecciones son conducidas en diferentes formatos, el costo de implementar una nueva modalidad, sin obtener un aumento en la participación o la inclusión no parece conveniente desde el punto de vista económico. ${ }^{39}$ Un elemento adicional que puede jugar en contra de la ventaja financiera del sufragio por Internet es su implementación allí donde la administración electoral está descentralizada como producto de una descentralización política del país. Mientras sería económicamente conveniente la implementación de un sistema centralizado, los costos de transacción entre diferentes agencias territoriales pueden suponer un incremento de costos disuasivo. Estas observaciones indican que las ventajas financieras del sufragio por Internet sólo podrían observarse en un sistema totalmente digitalizado sumado a una administración electoral centralizada. ${ }^{40}$

Los aspectos económicos no son los únicos que pueden ser promovidos por medio de un sistema de voto por Internet. En relación con los aspectos operacionales la principal ventaja es que el sufragio electrónico en general tiende a la producción de resultados mucho más rápidos y precisos que los sistemas manuales de conteo de votos, ${ }^{41}$ a lo que debe sumarse lo ya mencionado respecto a una considerable rebaja en la pérdida de votos respecto del sistema de sufragio por correo. También en cuanto a la mejora de aspectos operativos, la tecnología informática y el tiempo con que el votante cuenta cuando sufraga por Internet desde su hogar o su trabajo, pueden convertir el momento de la elección en una oportunidad que permita al elector informarse más sobre los candidatos y sobre qué está en juego en

38 Oostveen, Anne-Marie y Van den Besselaar, Peter, "Internet Voting Technologies...", cit., p. 64.

39 Ibidem, p. 74.

40 Beroggi, Giampiero, "Internet Voting: An...", cit., pp. 30-32.

41 Buchstein, Hubertus, "Online Democracy, is it Viable? Is it Desirable? Internet Voting and Normative Democratic Theory", en Kersting, Norbert y Baldersheim, Harald (eds.), Electronic Voting and Democracy: A Comparative Analysis, Springer, 2004, p. 45. 
la elección. Es perfectamente posible que el software de votación pueda contar con información adicional al nombre y el partido de los candidatos, incluyendo biografías, proyectos políticos, votaciones anteriores sobre temas controversiales, etcétera. Incluso si ese no es el caso, y el software de votación se limita a emular la información del voto en la papeleta de votación, el votante sigue contando con tiempo y e información para tomar una decisión más informada usando buscadores de Internet y la información pública disponible. Adicionalmente, la tecnología tendría el potencial de implementar sistemas más complejos de votación como repartir un voto entre dos candidatos o incluir una mayor complejidad en los plebiscitos haciendo que los ciudadanos puedan entregar un puntaje a cada opción en vez de elegir entre una $u$ otra con un sí o un no rotundo. ${ }^{42}$ Todos estos mecanismos podrían constituir de facto una inyección de democracia directa en un horizonte más amplio gobernado por la idea de representación.

Un punto adicional debe introducirse respecto a las complejidades económicas y operativas que puede significar la implementación adicional de un sistema de registro especial para votar por Internet. La hipótesis más simple en este supuesto es la de un sistema de sufragio totalmente electrónico que implique que no requiera un sistema especial de registro. Ello aplicaría tanto a casos en que el registro es automático como cuando el registro debe ser llevado a cabo por el ciudadano en orden de quedar habilitado para sufragar. Las cosas se complican considerablemente cuando ante la ausencia de un sistema electrónico de sufragio presencial, debe existir un sistema de registro paralelo para poder estar habilitado para sufragar por Internet. Esta complejidad adicional podría provocar un impacto negativo en el objetivo de facilitar el sufragio.

\section{Problemas DEL SUFRAGio POR CORREO E INTERNET}

\section{La afectación de la integridad de las elecciones}

La afectación de la integridad de la elección es sin lugar a dudas la principal razón que ofrecen los escépticos frente al sufragio por Internet y en este respecto presenta diferencias considerables con el sufragio por correo, que parece mucho más confiable. La noción de integridad electoral tiene

42 Idem. 
relación con el respeto de estándares internacionales que se aplican para juzgar las elecciones y que puede resumirse en la noción de llevar a cabo unas elecciones que resulten libres y justas. ${ }^{43} \mathrm{La}$ implementación práctica de un sistema que respete la integridad electoral requiere dar respuesta a los diferentes riesgos a los que un sistema de votación se expone con la informatización y con el hecho de que la votación se produce en contextos no controlados por las autoridades electorales. Se debe partir de la hipótesis de que los riesgos para la seguridad de la elección pueden minimizarse, pero nunca pueden eliminarse por completo. Por esto es que antes de eliminar todo riesgo, se puede sostener finalmente que de lo que se trata es de llegar a niveles de seguridad aceptables que permitan justificar la implementación de esta forma de sufragio conforme los beneficios en accesibilidad esperados. Los riesgos deben ser considerados con cuidado en la medida que pueden afectar la confianza pública en que las elecciones son efectivamente íntegras, lo que puede estar o no relacionado con que dichos riesgos se concreten y lleguen a cambiar el resultado de la elección.

Las brechas de seguridad o amenaza a la integridad de la elección pueden dar paso a dos tipos de problema. Por un lado, están los peligros de que los votos individuales puedan ser afectados, por ejemplo, mediante una suplantación de identidad del votante, por un virus computacional o por una falla de servicio provocada por un hacker que impida votar a un votante singular. Este problema primariamente afecta al votante individual quien se ve excluido de la elección, y aunque probablemente no tenga un impacto en el resultado final de la elección, de todas formas afecta el principio general de la igualdad electoral. En este aspecto, las hipótesis discutidas tienen similitudes con las vulnerabilidades que presenta el sufragio por correo. Por otro lado, y de manera mucho más dramática, están los peligros que amenazan el resultado general de la elección, por medio de una violación de la seguridad que permita, por ejemplo, intervenir en la integridad de elección a nivel de los datos electorales agregados, ya sea mediante una modificación de la información almacenada o mediante una falta de servicio sistémica que impida votar a un gran sector de la ciudadanía. Este problema afecta a todos los votantes, a los principios electorales más elementales y, por supuesto, afecta gravemente la confianza pública

43 Norris, Pippa, "Does the World Agree About Standards of Electoral Integrity? Evidence for the Diffusion of Global Norms", Electoral Studies, vol. 32, núm. 4, 2013, pp. 576-588. 
en que el resultado de la elección que es hecho público corresponde efectivamente con el resultado de la agregación de los sufragios efectivamente emitidos por los ciudadanos. Las amenazas de este tipo pueden venir tanto de las partes en disputa en la elección, candidatos y partidos, como del gobierno y de terceros sin interés en la disputa, como el caso de hackers. Este tipo de problema de seguridad no está limitado al sufragio por Internet, sino que se considera afecta a toda modalidad de sufragio electrónico donde la información electoral es procesada en forma digital sin respaldo físico, como es el caso del voto en papel. ${ }^{44}$

Para abordar este tipo de problemas es que un sistema de sufragio por Internet debe contar con ciertos requisitos. Ellos podrían sistematizarse bajo las demandas de verificabilidad, confidencialidad y transparencia. En primer lugar, un sistema de sufragio por Internet debe ser verificable (individual y universalmente). La verificabilidad individual consiste, por un lado, en que se debe asegurar un sistema que permita al votante y sólo al votante ejercer el derecho a sufragio que le corresponde. Normalmente esto estará asegurado con un proceso de autenticación adecuado. La combinación de dos elementos, como en el caso estonio (tarjeta de identificación más clave numérica), parece ser una opción conveniente. Por otro lado, cada votante debe tener la posibilidad de constatar que su voto ha sido efectivamente contado en el resultado electoral. La verificabilidad universal o auditabilidad consiste en que el diseño del sistema de votación debe permitir determinar, mediante auditorías y procesos de control ejercidos por el Estado, agencias autónomas o auditores externos, que los resultados de la votación coinciden con las preferencias efectivamente expresadas por los ciudadanos.

En segundo lugar, un sistema de sufragio por Internet debe resguardar la identidad del votante, esto es, debe ser confidencial. La posibilidad de vincular la identidad del votante con el contenido de su voto tiene toda una serie de inconvenientes que se asocian, por un lado, con el riesgo de coacción, fraude y venta de votos $\mathrm{y}$, por otro, con su utilización como una herramienta represiva de la oposición por parte de quienes están en el poder. Para satisfacer el requisito de confidencialidad, sin afectar el requisito de la verificabilidad, se requiere que nadie salvo el votante pueda identificar por quién votó. ${ }^{45}$

\footnotetext{
44 Buchstein, Hubertus, “Online Democracy, is...", cit., pp. 48 y 49.

45 Hall, Thad, "Internet Voting: the...", cit., p. 112.
} 
Finalmente, además de la verificabilidad y confidencialidad, un sistema de sufragio por correo debe ser transparente, esto es, debe permitir a la ciudadanía el control del proceso electoral llevado a cabo por el gobierno, sin afectar, por supuesto, la confidencialidad de la votación. De no ser transparente, los ciudadanos no tienen la menor posibilidad de saber si los resultados son legítimos o no. Esta fue exactamente el tenor de las dudas que expresó, en 2009, el Tribunal Constitucional Alemán cuando dictó una sentencia en que afirmó que el voto electrónico (en general) era incompatible con un método de votación confiable y que pueda ser comprendido por todo ciudadano. En concreto, afirmó que el artículo 38 de la Ley Fundamental Alemana, que establece el principio de publicidad electoral, era incompatible con el voto electrónico, en la medida que éste no garantizaba un control público por parte de la ciudadanía de los pasos esenciales del acto electoral y la determinación del resultado de manera fiable y sin conocimientos técnicos especiales. ${ }^{46}$

No obstante, al no existir evidencia relevante disponible sobre estos fenómenos, quienes abogan por el sufragio por Internet consideran que la adopción de medidas preventivas rigurosas debería disminuir los riesgos de afectación de la integridad de la elección a niveles bastante bajos. ${ }^{47}$ Es interesante mencionar que en algunos países donde se aplica el sufragio electrónico presencial, que está afecto a similares problemas en lo que respecta a la integridad de la elección, éste es considerado más fiable que el sufragio tradicional en papel. ${ }^{48}$

\section{La afectación del secreto del sufragio}

Un segundo aspecto que es visto como un problema es la afectación del secreto del sufragio. Si bien este problema se traslapa con la idea del requisito de confidencialidad propuesto más arriba, contiene elementos que necesitan ser tratados independientemente. Esta sección se refiere al secreto del sufragio frente a terceros diferentes de las autoridades que administran

46 Seedorf, Sebastian, "Germany: The Public Nature of Elections and its Consequences for E-Voting", en Mauer, Ardita y Barrat, Jordi (eds.), E-Voting Case Law: A Comparative Analysis, Burlington, Ashgate, 2006.

47 Gronke, Paul et al., "Convenience Voting...", cit., pp. 449 y 450; Oostveen, AnneMarie y Van den Besselaar, Peter, "Internet Voting Technologies...”, cit., p. 75.

48 Martínez Dalmau, Rubén, “Aspectos diferenciales del...”, cit., p. 234. 
y controlan la elección. En este sentido, el sufragio secreto se vería necesariamente afectado tanto por el sufragio por correo como por el sufragio por Internet, en la medida que tiene como finalidad impedir que terceros influencien mediante fraude, manipulación o coacción el voto de cada ciudadano y cuando el sufragio no se ejerce en un ambiente controlado por la autoridad, este tipo de relaciones puede generarse sin que sea percibido ni sea posible de evitar.

En la lógica que subyace al régimen de secreto que normalmente sigue al sufragio presencial, los votantes que han emitido su preferencia son libres de decir a cualquiera por quién han votado, sin embargo, no son libres de probarlo. Esa característica es considerada una garantía contra el fraude, la manipulación y la coacción toda vez que, no obstante la intervención de terceros puede suceder, éstos no pueden demandar una prueba de que efectivamente el voto se marcó con la preferencia deseada, para poner un ejemplo, por quien ha pagado con esa finalidad. ${ }^{49} \mathrm{El}$ secreto del sufragio, así, funciona obstruyendo la posibilidad de verificar si la acción que es incentivada por el pago o forzada mediante la amenaza es llevada a cabo $\mathrm{y}$, por tanto, eliminando los incentivos para, por ejemplo, la generación de un mercado de votos. Esta garantía es perturbada por el sufragio en ausencia, por correo y por Internet, ya que se hace imposible comprobar que el sufragio fue efectivamente realizado en secreto y, por tanto, ya no es posible eliminar la posibilidad de generar una prueba de que el sufragio marcó una preferencia concreta y se abre a la ocurrencia de toda esa serie de conductas que el secreto busca evitar. ${ }^{50}$

Si bien los sistemas electrónicos de verificación de identidad pueden perfeccionarse, simplemente no es posible imaginar un sistema que evite totalmente las hipótesis de abuso, dado que la ausencia de un control abrirá necesariamente la posibilidad de intervención por parte de terceros. Mediante las modalidades de sufragio en ausencia, la responsabilidad de asegurar el secreto del voto, y con esto la garantía de su integridad, son traspasadas desde el procedimiento público estatalmente controlado hacia el votante, que de esta manera, al mismo tiempo que se vuelve libre de pu-

49 Buchstein, Hubertus, "Public Voting and Political Modernization: Different Views from the Nineteenth Century and New Ideas to Modernize Voting Procedures", en Elster, Jon (ed.), Secrecy and Publicity in Votes and Debates, Cambridge, Cambridge University Press, 2015, p. 31.

50 Birch, Sarah y Watt, Bob, "Remote Electronic Voting: Free, Fair and Secret?", The Political Quarterly, vol. 75, núm. 1, 2004, pp. 60 y 65. 
blicitar su preferencia, se vuelve vulnerable a la interferencia de terceros. ${ }^{51}$ En la terminología de Buchstein, el sufragio por Internet sugiere un tránsito desde un sufragio total y obligatoriamente secreto hacia un sufragio opcionalmente secreto. ${ }^{52}$

En la medida que en el contexto del sufragio por Internet el secreto estará garantizado solamente por el ciudadano, es importante considerar si éste está en posición para evitar las intervenciones indebidas. Si bien podría partirse de la base de que todos los ciudadanos son suficientemente capaces de resistir la presión externa y que la integridad del sufragio no dependerá necesariamente de su secreto, la historia electoral ha demostrado que dicha expectativa no es sostenible..$^{53}$ Asimismo, es posible considerar varias hipótesis actuales en que, por ejemplo, las personas son objeto de presiones familiares para votar en cierto sentido, algo que es particularmente pernicioso en la medida que sabemos que en el contexto de la familia la igualdad no es el principio que organiza las relaciones entre miembros. ${ }^{54}$

Para disminuir dichos riesgos, podrían considerarse algunas medidas preventivas generales. En primer lugar, es conveniente que el sufragio por Internet se mantenga como una alternativa al sufragio presencial, ya sea éste en papel o electrónico. De esta manera, el ciudadano siempre contará con la posibilidad de sufragar en el secreto de la cámara de votación. En segundo lugar, sería conveniente permitir que quienes sufragan por Internet tengan la posibilidad de sufragar en sucesivas ocasiones y de esta manera que los votos posteriores anulen los votos anteriores. Asimismo, sería conveniente entregar la posibilidad para quienes hayan usado el sufragio por Internet que puedan volver a votar, esta vez de forma presencial y completamente secreta. Estas dos medidas deberían actuar contra la posibilidad de exigir una prueba de carácter definitivo de que se ha votado de una manera determinada. Sin embargo, ellas constituyen una protección para el elector frente a interferencia indeseada por parte de terceros y no

51 Buchstein, Hubertus, "Democracy's Secret: Carl Schmitt and the German Critique of Secret Voting", Teoksessa Redescriptions. The Finnish Yearbook of Political Thought, vol. 6, 2002, p. 108 .

52 Buchstein, Hubertus, "Online Democracy, is...”, cit., pp. 50-53.

53 Birch, Sarah y Watt, Bob, "Remote Electronic Voting...", cit., p. 65.

54 Ibidem, p. 69. 
eliminan la posibilidad de que el elector voluntariamente pueda vender su voto, someterse por su voluntad a un tercero o ser manipulado por éste. ${ }^{55}$

Es importante hacer una última reflexión acerca del este asunto. Incluso si se piensa que el secreto cumple una importante tarea democrática, éste no puede constituirse en un objetivo inderogable. En la medida que se busca proteger la participación electoral de ciudadanos vulnerables a través de su inclusión electoral mediante alguna forma como el sufragio en ausencia, este acceso puede erigirse en un objetivo tan importante que justifique una solución que considere un balance entre estas formas de proteger la participación democrática. Así, es posible considerar que un sistema de causales para solicitar el sufragio en ausencia resultaría en un adecuado sistema que considera ambos objetivos. ${ }^{56}$ Lo que en definitiva está en pugna, se ha sostenido, es el secreto del sufragio versus su universalidad, ${ }^{57}$ $\mathrm{y}$, de hecho, las formas ya existentes de sufragio presencial asistido son un ejemplo de compromiso entre el derecho a participar y el secreto del sufragio. Un estándar que puede definir esta posición de compromiso es el sugerido por Birch y Wattt: afectar el secreto del sufragio debe ser permitido en aquellos casos en que los votantes no podrían ejercer dicho derecho sin dicha afectación. ${ }^{58}$

\section{EL SIGNIFICADO DEL SUFRAGIO POR INTERNET}

Sin duda el cambio tecnológico, especialmente el que afecta la tecnología de las comunicaciones, ha impactado en cómo se conducen la política y las elecciones. La masiva influencia de la radio y la televisión durante el siglo XX es prueba de ello. ${ }^{59}$ En una sección anterior de este trabajo se mencionó que el concepto de democracia digital yacía en una zona gris entre la mejora de un modelo que en lo esencial permanece el mismo, y la superación de dicho modelo. Por un lado, la democracia digital supone la mejora de algunos aspectos procedimentales (seguridad, gestión, costos)

55 Saglie, Jo y Bock Segaard, Signe, "Internet Voting and...", cit., pp. 157, 159 y 60.

56 Fortier, John, Absentee and Early..., cit., p. 16.

57 Buchstein, Hubertus, "Public Voting and Political...", cit.; Saglie, Jo y Bock Segaard, Signe, "Internet Voting and...", cit., p. 157.

58 Birch, Sarah y Watt, Bob, "Remote Electronic Voting...", cit., p. 64.

59 Alvarez, R. Michael y Nagler, Jonathan, "Likely Consequences of...", cit., p. 1115 . 
del modelo representativo y eventualmente la incorporación de elementos que incrementarán su legitimidad (más deliberación y participación) en términos de los propios estándares democráticos. Por otro lado, la democracia digital parece movernos a la superación del modelo representativo en términos, por ejemplo, de un modelo de gobierno postmoderno. ${ }^{60} \mathrm{Si}$ bien el sufragio por Internet no va constituir en sí mismo este cambio de paradigma, éste podría ser un indicador de la dirección que estamos tomando.

En la búsqueda de esos indicios, en esta última sección del trabajo se elaboran, sobre la base de la hipótesis de implementación de un sistema de sufragio por Internet, algunas observaciones que apuntan a cambios en la concepción tradicional de las elecciones. ${ }^{61}$ Ya se ha mencionado el cambio que implicaría que la votación no fuera estrictamente secreta. Se pueden considerar tres aspectos que también forman parte de este paradigma tradicional (sufragio presencial, simultáneo y con cierto costo para el votante) que han sido cuestionados por formas de sufragio no presencial y que podrían ser cuestionados aún en forma más intensa por la introducción del sufragio por Internet.

\section{Juntos en el espacio público}

En primer lugar, algunos autores han llamado la atención sobre el efecto que la adopción de modalidades no presenciales de sufragio tiene sobre muestra comprensión de la jornada electoral. Al ausentarse de los locales de votación, los ciudadanos pierden la oportunidad de experimentar el ritual comunitario de votar en conjunto. ${ }^{62}$ Ese ritual tiene aspectos simbólicos, pero también aspectos prácticos. Entre los primeros, está la cuestión simbólica de la igualdad que es asociada al encuentro de las personas en el espacio público en su calidad de ciudadanos libres e iguales. Como lo sostiene Dennis Thomson,

cuando los ciudadanos van a las urnas durante el mismo día, participando públicamente en una experiencia común de compromiso cívico, demues-

60 Barrientos del Monte, Fernando, "Dimensiones discursivas en torno al voto electrónico”, Revista de Ciencia Política (Santiago), vol. 27 núm. 1, 2007, pp. 111-131; Kersting, Norbert y Baldersheim, Harald, "Electronic Voting and...”, cit., pp. 14-16.

61 Orr, Graeme, Ritual and Rhythm..., cit., p. 54.

62 Ibidem, p. 58. 
tran su voluntad de participar en el proceso democrático en pie de igualdad. Ir al local de votación y pararse en la fila junto a sus vecinos puede no ser uno de los momentos más excitantes de la vida, pero el día de la elección sirve como un importante propósito expresivo de la democracia. Permite a los ciudadanos para expresar su compromiso a un proyecto común en que participan en pie de igualdad. ${ }^{63}$

Además, la adopción del sufragio por Internet tiene el potencial de privatizar el proceso electoral en la medida que el lugar donde éste se realiza pierde importancia y, por tanto, deja de aportar su carga simbólica. El evento de la elección se traslada desde el espacio público hasta el espacio privado del hogar o del lugar de trabajo quedando divorciado del que simboliza la preocupación por el bien común, transformando también, probablemente, la forma en que los ciudadanos experimentan su participación en dirección a la "erosión del espíritu de votación pública, un cambio en la cultura política, y un apoyo a la tendencia de la privatización de la política". ${ }^{64}$ Incluso, puede pensarse esta pérdida del carácter público como una trasformación que se dirige a entender el acto de votar como una experiencia de consumo, centrada en la satisfacción del cliente. ${ }^{65}$

Por el contrario, se puede argumentar que el concurrir a las urnas es más que un mero ritual e implica involucrarse en un ambiente que permite, en la práctica, desarrollar la educación cívica y democrática de los votantes. En este sentido, la privación de la experiencia común de participación presencial puede producir, más que mayor participación, apatía electoral y la inhibición de la capacidad del ciudadano de sentir que es parte de algo más grande, de una comunidad política. Esto último puede significar entrar en el terreno de la sicología política, pero esta intromisión parece justificada si es que en verdad no podemos prescindir de la experiencia presencial para entender el sentido de pertenencia que es clave en una acción colectiva. Asimismo, la participación presencial constituye una comprobación por los sentidos de la transparencia e integridad de los resultados. ${ }^{66}$ Así, si el voto por Internet tiene la capacidad de destruir esos rituales cívicos

63 Thompson, Dennis, "Election Time: Normative Implications of Temporal Properties of the Electoral Process in the United States", American Political Science Review, vol. 98, núm. 1, 2004, p. 58.

64 Buchstein, Hubertus, “Online Democracy, is...", cit., p. 55.

65 Orr, Graeme, Ritual and Rhythm..., cit., p. 64.

66 Ibidem, pp. 65 y 66. 
tan importantes para la conservación de una cultura política participativa, que incluye tanto elementos simbólicos como prácticos, quizás sea sensato mantenerse alejado de él. ${ }^{67}$

Sin embargo, no todos quienes piensan en el sufragio no presencial desde el punto de vista de la preservación o generación de una cultura política participativa coinciden en este diagnóstico. Richey ha defendido el efecto que podría tener el sufragio en ausencia para el aumento de la discusión política. ${ }^{68} \mathrm{Su}$ argumento consiste en afirmar que un voto que no es secreto puede ser transformado en una experiencia compartida con las personas más cercanas, familia y amigos, incentivando de esta manera la discusión política mientras se determina cómo se votará. De esta manera, desestima la afirmación de que la experiencia de votar en ausencia es una experiencia que desvincula al votante de la experiencia cívica y democrática de la elección. Por el contrario, como sugiere la investigación de Richey, la mayor discusión política propiciada por el sufragio en ausencia podría generar mayor involucramiento político y eventualmente acrecentar la participación electoral por esa razón. En contraste, sostiene que la interacción social que involucra concurrir al recinto de votación es limitada y no tiene un potencial deliberativo, como ya lo había pensado siglos antes John Stuart Mill. ${ }^{69}$

\section{Votar al mismo tiempo}

El segundo aspecto que puede vincularse a las formas de votación no presencial es que terminan con el principio de la simultaneidad de la elección, esto es, la idea conforme a la que todos los participantes en la elección votan más o menos al mismo tiempo. Conforme a Thomson, que todos los votantes sufraguen al mismo tiempo es importante por dos razones. Primero, porque mientras más concentrada en el tiempo, la elección tiene más potencial para expresar la voluntad de una determinada mayoría. La simultaneidad, crea, en este respecto una soberanía popular más coherente. Segundo, y más importante conforme a Thomson, la simultaneidad es

67 Oostveen, Anne-Marie y Van den Besselaar, Peter, "Internet Voting Technologies...", cit., p. 75 .

68 Richey, Sean, "Who Votes Alone? The Impact of Voting by Mail on Political Discussion”, Australian Journal of Political Science, vol. 40, núm. 3, 2005, pp. 435-442.

69 Véase Buchstein, Hubertus, "Online Democracy, is...", cit. 
importante porque es compatible con el valor de la equidad democrática. Esto último significa, en el contexto que nos ocupa, que todos los participantes cuenten con la misma información al sufragar. ${ }^{70}$

El sufragio por Internet y otras formas de sufragio no presencial tienden a cuestionar el principio de simultaneidad. Normalmente, para el ejercicio de estas formas de sufragio se establece un periodo de tiempo previo a la elección que puede fluctuar entre un mes y una semana; así, los votantes tienen mayores oportunidades para sufragar. Ello implica dos problemas: uno real y otro virtual.

El primero se refiere a la afectación de las campañas electorales por la pérdida de simultaneidad. Un aspecto importante de la simultaneidad de la elección es que permite diseñar de mejor manera la forma en cómo los electores van a ser destinatarios de las campañas electorales. Cuando el sufragio en ausencia es permitido durante un periodo de tiempo variable con anterioridad al día de la elección, los votantes toman sus decisiones sin tomar en consideración sucesos importantes que acontecen en el periodo de la campaña. ${ }^{71}$ El rol presuntamente deliberativo de la etapa de campaña electoral, en que diversos mensajes deben ser comunicados y discutidos, puede ser así tremendamente afectado. Asimismo, esto lleva a que las campañas electorales cambien sus estrategias para poder alcanzar a aquellos votantes que sufragan con anterioridad al día de la elección. Esto puede tener un impacto importante en el aumento del gasto electoral a niveles que podrían alcanzar el $25 \%{ }^{72}$ Todo esto lleva a pensar que, desde la perspectiva del voto en ausencia, cuando éste se abre por un periodo de tiempo suficientemente extenso antes de la votación, se asume implícitamente que las campañas electorales no son importantes y que la elección gira sólo en torno al acto individual de emitir preferencias, desconectado del ritual complejo que involucran las elecciones en democracia. ${ }^{73}$

El segundo problema, que consiste en un problema virtual vinculado a la pérdida de simultaneidad, es que los resultados provisionales de las votaciones por Internet podrían estar disponibles para aquellos que todavía no han sufragado, afectando así el principio de la equidad democrática

70 Thompson, Dennis, "Election Time: Normative Implications...”, cit., pp. 56-59.

71 Gronke, Paul et al., "Convenience Voting”, cit., p. 445; Fortier, John, Absentee and Early Voting..., cit., p. 62.

72 Gronke, Paul et al., "Convenience Voting", cit., p. 445.

73 Orr, Graeme, Ritual and Rhythm in Electoral Systems..., cit., p. 62. 
formulado por Thomson. Esto podría llevar a un cambio en la forma en que sufragamos, esto es, un modo que, por sobre las propias convicciones políticas, presta atención a cómo los otros sufragan y toma en cuenta cuál será el impacto de mi voto en el resultado. La ocurrencia de este problema, por supuesto, depende de si se toma la decisión de publicitar los resultados parciales de la votación por Internet en vez de mantenerla en secreto hasta que los recintos de votación presencial hayan cerrado. Esto parece poco sensato si se atienden los problemas similares que otra práctica similar - como son las encuestas a boca de urna - han generado en países como los Estados Unidos. Allí, los votantes de la costa oeste son disuadidos o persuadidos de ir a votar por los resultados preliminares y no oficiales que son informados por la televisión de lo que está ocurriendo en la costa este. $^{74}$

Debe considerarse, sin embargo, que el sufragio por Internet no tiene por qué necesariamente afectar la simultaneidad de la elección. Si bien este es el caso del sufragio por correo, y hasta ahora ha sido el caso de las experiencias de sufragio por Internet, el periodo para sufragar podría ser limitado a un plazo más corto y eventualmente a un solo día que coincidiera con el día de la votación presencial. No hay nada en el sufragio por Internet que impida esa posibilidad.

\section{El valor cívico de un sufragio costoso para el votante}

Una tercera característica que parecería ser afectada o transformada por la implantación del sufragio por Internet es el valor cívico del sufragio. En el régimen de sufragio presencial, el elector tiene que hacer un esfuerzo (razonablemente limitado) para concurrir a la urna de votación. Ese esfuerzo implica, o da cuenta, de que dicha actividad es valiosa y que contribuye a producir algo de lo que todos, como miembros de la comunidad, disfrutamos. Cuando se señala que uno de los principales objetivos de la implantación del sufragio por Internet es incrementar la participación electoral mediante la facilitación del acceso al sufragio, se está partiendo de la base que los ciudadanos no tienen incentivos para participar y que dicha participación, incluso cuando consiste solamente en concurrir a votar una vez cada cuatro años, es muy costosa para su plan de vida individual.

\footnotetext{
74 Thompson, Dennis, “Election Time: Normative Implications...”, cit., pp. 58 y 59.
} 
La pregunta que se sigue de este razonamiento es si dicha participación electoral adicional podría mejorar la calidad de nuestras prácticas democráticas y aquí hay opiniones que consideran que la facilitación como horizonte normativo del sufragio no presenta perspectivas auspiciosas. Buchstein, por ejemplo, sostiene que esta aproximación podría llevar a la implementación de una democracia directa consistente sólo en apretar botones, dando un impulso tecnológico al populismo imperante en nuestros días. ${ }^{75}$ Asimismo, implica que un conjunto de personas sin ningún interés por las decisiones políticas tendría acceso a tomar decisiones frívolamente espontáneas, reduciendo de esta manera el valor del sufragio a tal extremo que quizás sea mejor para la democracia que dichos votantes se mantengan fuera de la actividad electoral. Si de verdad existe una preocupación por aumentar la participación electoral, persuadiendo a los votantes desinteresados, la solución, sin duda, no puede pasar por transformar las elecciones en una especie de juego electrónico en que los nuevos votantes sean incorporados por el atractivo tecnológico, sino en propiciar un contexto de actividad política que provoque de parte de los ciudadanos un real interés por participar en la cultura política que es condición de posibilidad y que justifica normativamente las elecciones.

Si bien estas razones para evitar la facilitación de la participación electoral deben tomarse en cuenta, ellas no pueden transformarse en un impedimento para la implementación de un sistema mediante el cual aquellos ciudadanos que son excluidos de facto de la elección, puedan acceder al sufragio. Los casos más obvios son los de los ciudadanos en el extranjero (temporal o permanentemente, cuando en este último caso ellos conservan su derecho a sufragio), las personas con una discapacidad motora, los adultos mayores con dificultades de desplazamiento, los enfermos postrados, las personas con responsabilidades en el hogar, etcétera. Si su inclusión implica la necesidad de adoptar un modelo de sufragio por Internet (o por correo) que demande la expresión de excusas por parte del votante o si, por el contrario, implica la adopción de un modelo de sufragio a distancia disponible para todos los ciudadanos, es una cuestión a considerar cuidadosamente.

75 Buchstein, Hubertus, “Online Democracy, is...”, cit., p. 55. 


\section{CONCLUSIÓN}

La aproximación a la tecnología para solucionar algunos problemas que afectan a la democracia representativa puede llevar, sin que estemos necesariamente consciente de ello, a la modificación de las prácticas democráticas como las entendemos hoy en día. El caso de la participación electoral vía Internet, como mecanismo de facilitación e inclusión, puede dar paso a un proceso transformativo que abra la puerta a la incorporación de formas de la democracia directa y la administración democrática descentralizada, asociadas a formas progresistas de la democracia digital. Hasta ahora, sin embargo, el debate sobre el uso de la tecnología virtual para el ejercicio del sufragio ha estado centrado en las ventajas y desventajas que esta modalidad presenta por sobre las modalidades tradicionales de votación presencial y por correo.

Respecto a las ventajas, si bien las otras formas de sufragio en ausencia parecen no haber promovido los niveles de participación, y contribuyendo a veces a desincentivar la participación de sectores desaventajados, el sufragio por Internet tiene el potencial de subsanar algunas de estas deficiencias, especialmente cuando se tiene cuenta que las nuevas tecnologías en red son cada vez más accesibles. Por otro lado, el carácter inclusivo del sufragio por Internet tiene el potencial de perfeccionar la verdadera ventaja que otras formas de sufragio no presencial tienen sobre el sufragio presencial. El acceso por esta vía de personas que no tienen la posibilidad de sufragar presencialmente, vistos los problemas del sufragio por correo, hace de su implementación más un asunto de justicia con ciertas minorías que un asunto de aumento de la participación electoral general. Por último, si bien la administración electoral se haría más fácil, eficiente y barata, un sistema de votación por Internet tendría un costo de implementación considerable, especialmente tomando en cuenta que existen buenas razones para mantener transitoria pero también permanentemente un sistema de votación presencial.

Respecto a los problemas: si bien existen legítimas dudas respecto de la afectación que un modelo de sufragio por Internet podría generar en la integridad de las elecciones, los riesgos no son considerablemente mayores que aquellos generados por otros modelos electrónicos de votación, que son comunes en el derecho comparado y que parece ser la tendencia en términos de modalidades de votación. La demanda por un sistema de 
escrutinio comprensible y público de las elecciones, es quizás la más importante objeción que ningún sistema totalmente electrónico está en posición de satisfacer. Por otro lado, la afectación del secreto del sufragio es un tema bastante complicado y sin duda requiere una investigación más detallada. Si bien el riesgo de fraude, coacción o manipulación es serio, por otro lado, la inclusión de votantes hace necesaria, por razones de justicia, la implementación de un sistema de sufragio en ausencia. La introducción del sufragio por Internet se justificaría, así, para aquellos casos en que un problema impide al elector concurrir a votar presencialmente y no como una manera de facilitar el acceso general al sufragio.

En conclusión, no existen razones concluyentes ni para oponerse ni para buscar la implementación del sufragio por Internet a toda costa. Las consideraciones más importantes parecen ser aquellas que se refieren al cambio en la comprensión simbólica del sufragio en el contexto de la democracia representativa. Que el concurrir a sufragar conjunta y simultáneamente en el espacio público pierda el lugar central que hoy tiene en la experiencia democrática constituye quizás la pregunta más importante que surge del análisis de las consecuencias que el sufragio por Internet acarrearía.

Como una nota crítica, cabe juzgar las propuestas de quienes buscan aumentar la participación mediante la implementación del sufragio por Internet de irresponsables, cuando culpan a los métodos de votación por la alta abstención, antes de mirar a los déficits representativos de sistema político. Se trata de preguntarse por qué los ciudadanos no votan y no a pensar cómo votan. La información que tenemos es que la tecnología no aumentará la participación, de hecho algunos sugieren que ésta podría incluso disminuir como consecuencia de la virtualización electoral. Por el contrario, existe evidencia abrumadora que la participación aumentará con mayor información, movilización y motivación de los electores, los que constituyen elementos fundamentales de una cultura política y electoral robusta y que sí pueden transformar las actuales tendencias de disminución de la participación. ${ }^{76}$

No obstante estas dudas, en la medida que el uso de tecnología se acreciente, y las barreras tecnológicas tiendan a desaparecer, el proceso de incorporación del Internet en la práctica electoral se hará más probable al mismo tiempo que más natural. Por supuesto que la cultura política

76 Oostveen, Anne-Marie y Van den Besselaar, Peter, "Internet Voting Technologies...", cit., p. 65 . 
y electoral de cada país debe ser tomada en consideración a la hora de pensar en implementar un sistema tal, porque lo que funciona para ciertos países puede no funcionar para otros. No obstante esto, la introducción del sufragio por Internet es preferible frente a la introducción del sufragio por correo, para aquellos países que no hayan todavía desarrollado una modalidad de sufragio en ausencia.

Fecha de recepción: 3 de abril de 2017.

Fecha de dictamen: 8 de septiembre de 2017. 